\title{
Research
}

Carl Llor, Jordi Madurell, Montse Balagué-Corbella, Mónica Gómez and Josep Maria Cots

\section{Impact on antibiotic prescription of rapid antigen detection testing in acute pharyngitis in adults:}

\author{
a randomised clinical trial
}

\begin{abstract}
Background

Acute pharyngitis is one of the most frequent reasons for a GP consultation, and in most cases an antibiotic is prescribed.

Aim

To determine the impact of rapid antigen detection testing (RADT) to identify group $A$ beta haemolytic streptococcus in acute pharyngitis on the utilisation of antibiotics and appropriateness of their use.

\section{Design and setting}

Cluster randomised controlled trial in primary care centres in Catalonia, Spain.

\section{Method}

Patients with acute pharyngitis aged 14 years or older with at least one Centor criterion (fever, tonsillar exudate, tender enlarged anterior cervical lymph nodes, or absence of cough were recruited. Participant physicians were randomly assigned to one of two study arms: an intervention group (assigned to RADT) and a control group (following usual care, without RADT).

\section{Results}

Of the 557 adults enrolled, 543 could be evaluated for analysis (281 [51.7\%] in the intervention group and 262 [48.3\%] in the contro group). GPs without access to RADT were more likely to prescribe antibiotics compared with those who performed rapid tests $164.1 \%$ versus $43.8 \%, P<0.0011$. The more Centor criteria the patients presented, the greater the number of antibiotics prescribed, regardless of whether RADT was available $(P<0.001)$. Antibiotics were prescribed in $30.7 \%$ of the cases with negative RADT results. Inappropriate antibiotic

\section{INTRODUCTION}

Acute pharyngitis is a frequent cause of visits and antibiotic prescribing in the primary care setting. It has been estimated that $52 \%$ to $89 \%$ of adults with acute pharyngitis are treated with antibiotics. ${ }^{1-7}$ However, group $A$ beta haemolytic streptococcus (GABHS) is the cause in only $10-30 \%$ of the cases of acute pharyngitis, and is even less frequent in adults, accounting for $10-15 \%$ of all the infections. ${ }^{7.8}$ The majority of authors recommend the use of antibiotics for streptococcal pharyngitis, as prompt antibiotic therapy is associated with a slightly faster recovery of symptoms, prevention of early and late complications, and reduced spread of the strain to others. ${ }^{9}$ In the UK, the National Institute for Health and Clinical Excellence (NICE) recommends that clinicians consider immediate treatment with antibiotics for patients who have three or more Centor criteria: fever, tonsillar exudate, tender enlarged anterior cervical lymph nodes and absence of cough. ${ }^{10}$ However, regardless of whether they receive treatment with antibiotics, $85 \%$ of patients with acute pharyngitis are completely free of symptoms after the first week. ${ }^{11}$ A study of patients with tonsillitis in 17 European countries found that the mean duration of fever was 2-3 days, irrespective of the use of an antibiotic. $^{12}$ In addition, serious complications are uncommon nowadays. ${ }^{13,14}$
\end{abstract}

prescription was observed in 226 cases (43\%), and was significantly greater in the control than in the intervention group $160 \%$ versus $26.9 \%$; $P<0.001)$.

\section{Conclusion}

Even though more than 30\% of negative RADT results resulted in antibiotic prescribing, the study findings support the use of RADT in the consultation. This strategy has an important impact on reducing antibiotic prescription among adults with acute pharyngitis.

\section{Keywords}

criteria, clinical; clinical trials, randomised rapid antigen detection test; primary care; Streptococcus; Streptococcus Group A.
C Llor, MD, family physician, Primary Healthcare Centre Jaume I, Tarragona, University Rovira I Virgili, Spain. J Madurell, MD, family physician, Primary Healthcare Centre Breda-Hostalric, Institut d'Assistència Sanitària de Girona, Universitat Autònoma de Barcelona, Spain. M Balagué-Corbella, MD, family physician, Centre Penitenciari d'Homes, Catalonian Institute of Health, Barcelona, Spain. M Gómez, MD, family physician, Primary Healthcare Centre Doctor Josep Torner i Fors, Salut Maresme, Malgrat de Mar, Spain. JM Cots, MD, family physician, Primary Healthcare Centre La Marina, Barcelona, University of Barcelona, Spain.
Overuse of antibiotics can lead to side effects and the emergence of antibiotic resistance. ${ }^{15}$ The main reason for antibiotic overprescription in acute pharyngitis is the difficulty of obtaining a rapid and correct aetiological diagnosis. In addition, physicians often believe that patients expect an antibiotic and, in case of doubt, GPs are more prone to prescribing antibiotics.

In many countries, including Spain, rapid antigen detection tests (RADTs) are seldom used in general practice and physicians still rely on the Centor criteria for prescribing antibiotic therapy. ${ }^{16}$ However, due to the fact that these findings are non-specific and are commonly found in cases of viral origin, even experienced physicians may accurately diagnose streptococcal pharyngitis based on the clinical findings alone in no more than $75 \%$ of the cases..$^{17}$ In addition, primary care physicians evaluate each of the Centor criteria differently. Thus, in Spain, physicians are 28-fold more inclined to prescribe an antibiotic in acute pharyngitis when this is accompanied by tonsillar exudates. ${ }^{2}$ Moreover, in the different clinical trials carried out, the use of sore throat decision rules based only on clinical criteria have not been shown to be useful in reducing the prescription of antibiotics. ${ }^{18-20}$

Culture of a throat swab has been the standard method for diagnosing streptococcal pharyngitis. However, the

Address for correspondence

Carl Llor, c. Foixarda, 95. 43008 Tarragona, Spain.

E-mail: carles.llordurv.cat

Submitted: 1 August 2010; Editor's response:

3 September 2010; final acceptance:

22 October 2010.

CBritish Journal of General Practice

This is the full-length article (published online

26 Apr 2011) of an abridged version published in print. Cite this article as: $\mathbf{B r} \mathbf{J}$ Gen Pract 2011; DOI: 10.3399/bjgp11X572436 


\section{How this fits in}

This is a clinical trial comparing the proportion of antibiotic prescription and the inappropriateness of this prescription for suspected acute pharynigitis among two groups of physicians: GPs in one group were provided with rapid antigen detection tests as a guide for prescribing antibiotics in adults and at least one clinical criterion of streptococcal aetiology; GPs in the second group followed their usual clinical practice, without the use of these tests, to decide whether treatment should include antibiotics. Results of the study demonstrate that the intervention was effective in significantly decreasing antibiotic prescription by more than $20 \%$ and in reducing the inappropriateness of prescription by $33 \%$. However, this reduction would probably have been greater if physicians who were randomly assigned to the intervention group had trusted the results of these rapid tests, indicating that training aimed at physicians who have never used these tests is essential.

delay in obtaining results makes its performance useless in clinical practice. Over recent years, low-cost and rapid immunological techniques targeting the detection of GABHS antigens have been developed for use in the clinical setting. These tests offer the advantage of diagnosing streptococcal pharyngitis within a few minutes, with an associated specificity of more than $95 \%$ and a sensitivity ranging from $60 \%$ to $96 \%$, using culture as the gold standard method. ${ }^{21,22}$ Several papers have shown that the use of these tests can reduce the prescription of antibiotics for pharyngitis in children. However, very few studies have examined the impact of RADT on the pattern of how GPs prescribe antibiotics for adult patients with pharyngitis. ${ }^{20,23,24}$ This randomised clinical trial was carried out to compare the percentage of antibiotic prescription and the inappropriateness of antibiotic prescription by GPs using RADT and GPs whose antibiotic prescription was only based on clinical criteria.

\section{METHOD}

The methodology of this study has been described in detail elsewhere. ${ }^{25}$ In brief, this was a cluster multicentre parallel randomised clinical trial carried out in 20 primary healthcare centres in Catalonia, Spain from January to May 2008. No blinding techniques were used. All physicians were asked to recruit consecutively the first 10 patients aged 14-60 years diagnosed with acute pharyngitis with one or more Centor criteria: fever, sore throat, tonsillar exudate, tender cervical nodes, and/or absence of cough. All patients were asked to provide written informed consent. Exclusion criteria were patients with more than five episodes of pharyngitis over the last year; those with immunosuppressed condition, such as active neoplasm, acquired immunodeficiency syndrome, or reception of chemotherapy, radiotherapy, steroids, and/or immunosuppressive therapy; those with heart valve disease; rheumatic fever; an episode of pharyngitis treated with antibiotics in the previous 15 days; and those who had tonsillectomy.

Participating primary healthcare centres were randomised to the intervention or to the control arm of the study, with an allocation ratio of $1: 1$, by a random sequence generated by a computer program. Physicians allocated to the intervention group were provided with RADT and those assigned to the control group managed streptococcal pharyngitis with only clinical criteria. Samples were taken by GPs who had been previously trained to perform the technique correctly with vigorous rotation of the tonsils and the posterior pharynx without touching the tongue, teeth, or gums. RADTs were undertaken with the $\mathrm{OSOM}^{\circledR}$ Strep A test (Genzyme) following the manufacturer's instructions. All physicians participating in this study sent a pharyngeal swab for culture. These samples were sent to the Department of Microbiology of the two hospitals of the area, with AMIES (Copan Innovation, Italy) as medium. Samples were seeded on a plate of blood agar and incubated at $37^{\circ} \mathrm{C}$ in an atmosphere of $\mathrm{CO}_{2}$ at $5 \%$ over 48 hours. A culture was considered positive for GABHS with a growth of any number of beta-haemolytic colonies, Gram staining with streptococcal morphology, and a catalase-negative test with posterior identification with an automated panel for WIDER Gram-positive cocci (Soria Melguizo). Results were confirmed with posterior serogrouping with the Streptococcal Grouping Kit (Oxford, UK). The culture was considered negative after 
48 hours of incubation with the absence of beta-haemolytic colonies.

The participating physicians collected the variables in a data registry notebook and reported age, sex, clinical symptoms (fever, sore throat, tender cervical nodes, absence of cough, abrupt onset), culture result, use of RADT or not, antibiotic or not, type of antibiotic, and evolution within the first month. Primary outcomes were the percentage of antibiotic prescription and the proportion of inappropriate antibiotic prescription. The latter included both patients without GABHS infection treated with antibiotics and those with GABHS infection in which antibiotic therapy was not given. Secondary outcomes were the type of antibiotics prescribed, the validity of the RADT, and full clinical recovery at the third week. The latter included patients without side effects, visits that required a change of treatment, or the presence of complications. For calculating the sample size, it was considered that 276 patients were required in each arm to detect a reduction in antibiotic prescription from $85 \%$ in the control group to $75 \%$ in the intervention group with a power of $90 \%$ and a level of significance of $5 \%$ (two-sided), allowing 10\% for losses in the follow-up visits.

Data were analysed in accordance with CONSORT guidelines. Descriptive statistics of the outcome measures were performed, as well as baseline characteristics and clinical measures, calculating means and standard deviations or percentages; $\chi^{2}$ tests were carried out to assess the impact of RADT on the percentage of antibiotic prescription and its inappropriateness in the intervention and control arms. Only $P_{-}$ values $<0.05$ were considered statistically significant.

\section{RESULTS}

Of the 80 physicians invited to participate, 19 declined (Figure 1). The number of cases included ranged from six to 10 per physician. During the period of study, 557 adults with acute pharyngitis and at least one Centor criterion were enrolled by 61 GPs. Of these, data for 543 were analysed (281 [51.7\%] in the intervention group and 262 [48.3\%] in the control group). Table 1 describes the demographic data and the clinical criteria according to the group assigned. No statistically significant differences were found in these variables in either group. Of the physicians assigned to RADT, these rapid tests were performed in 280 cases $(99.6 \%)$, while rapid tests were performed in five cases $(1.9 \%)$ in the control group. Antibiotics were prescribed in 291 cases $(53.6 \%)$, with amoxicillin being the most frequently prescribed in 154 cases (52.9\%), followed by penicillin in 58 (19.9\%), amoxicillin plus clavulanic acid in 51 (17.5\%), and macrolides in 20 cases $(6.8 \%)$. Other antibiotics were prescribed in nine patients (3.1\%). In seven cases, allergy to penicillin was reported.

GPs allocated to the control group prescribed antibiotics in 168 cases out of a total of 262 (64.1\%), significantly more than the physicians assigned to the intervention group (123 of 281 [43.8\%]; $P<0.001$ ). The greater the number of Centor criteria presented by the patients, the more antibiotics were prescribed regardless of whether RADT was available, although antibiotic prescription was significantly
Patients in whom antibiotics were given ( $n=123 ; 43.8 \%$ ) 


\begin{tabular}{|c|c|c|c|c|}
\hline Characteristic & $\begin{array}{l}\text { Intervention group } \\
\quad(n=281)^{\mathrm{a}}\end{array}$ & $\begin{array}{l}\text { Control group } \\
(n=262), a\end{array}$ & $\begin{array}{c}\text { Total } \\
(n=543)^{a}\end{array}$ & $P$-value \\
\hline Male & $103(36.7)$ & $99(37.8)$ & 202 (37.2) & 0.404 \\
\hline Mean age, (SD) years & $31.8(11.5)$ & $31.5(11.4)$ & $31.7(11.4)$ & 0.688 \\
\hline Fever & 199 (70.8) & 190 (72.5) & $389(71.6)$ & 0.533 \\
\hline Tonsillar exudate & $141(50.2)$ & $140(53.4)$ & 281 (51.7) & 0.379 \\
\hline Tender cervical lymph nodes & $114(40.6)$ & 106 (40.5) & 220 (40.5) & 0.942 \\
\hline Absence of cough & $211(75.1)$ & $195(74.4)$ & 406 (74.8) & 0.977 \\
\hline Abrupt onset & $126(44.8)$ & $109(41.6)$ & 235 (43.3) & 0.952 \\
\hline Number of Centor criteria & & & & 0.898 \\
\hline 1 & $70(24.9)$ & $61(23.3)$ & 131 (24.1) & \\
\hline 2 & $89(31.7)$ & $82(31.3)$ & $171(31.5)$ & \\
\hline 3 & $76(27.0)$ & $74(28.2)$ & $150(27.6)$ & \\
\hline 4 & $46(16.4)$ & $45(17.2)$ & $91(16.8)$ & \\
\hline
\end{tabular}

\section{Table 2. Prescription of antibiotics by group, according to number of} Centor criteria

\begin{tabular}{|c|c|c|c|}
\hline $\begin{array}{l}\text { Number of } \\
\text { Centor criteria }\end{array}$ & $\begin{array}{l}\text { Intervention group } \\
(N=281), n(\%)\end{array}$ & $\begin{array}{l}\text { Control group } \\
(N=262), n(\%)\end{array}$ & $\begin{array}{c}\text { Total } \\
(N=543), \mathrm{n}(\%)\end{array}$ \\
\hline 1 & $4(5.7)$ & 20 (32.8) & 24 (18.3) \\
\hline 2 & $29(32.6)$ & 48 (58.6) & 77 (45.0) \\
\hline 3 & 53 (69.7) & 57 (77.0) & 110 (73.3) \\
\hline 4 & 37 (80.4) & $43(95.6)$ & 80 (87.9) \\
\hline Total & 123 (43.8) & $168(64.1)$ & $291(53.6)$ \\
\hline
\end{tabular}

greater among physicians assigned to the control group $(P<0.001)$ (Table 2)

Cultures were performed in 526 patients with pharyngitis. Of these, the culture was positive for GABHS in 88 cases (16.7\%). Among physicians assigned to the intervention group, the culture was positive for GABHS in 49 cases (18.1\%), while it was positive in 39 patients (15.3\%) in the control group, with no statistically significant differences observed between the two groups. The rapid test was positive in 60 cases $(21.1 \%)$. In these cases, antibiotics were prescribed in 59 patients (98.3\%). On the other hand, antibiotics were prescribed in 69 of the 225 patients in whom the test for GABHS was negative (30.7\%). The greatest proportion of antibiotics prescribed in cases of negative RADT was observed among patients with four Centor criteria; thus antibiotics were prescribed in 21 out the 29 RADT-negative cases (72.4\%). The other percentages of antibiotic prescription in cases that were RADT negative were $57.4 \%$, $18.6 \%$, and $5.8 \%$ in patients with three, two, and one Centor criteria respectively. As shown in Table 3, the sensitivity of RADT was $89.8 \%$, with a specificity of $93.8 \%$.
Treatment was inappropriate in 226 cases $(43 \%)$. Of these cases, antibiotic treatment was not necessary in 210, and in 16 more the culture was positive for GABHS and antibiotic treatment was not prescribed. The inappropriateness of the antibiotic treatment was significantly greater among physicians assigned to the control group (153 cases; 60\%) than in the intervention group (73 cases; $26.9 \%$; $P<0.001$ ). Likewise, the greater the number of Centor criteria presented by the patient, the greater the inappropriateness of prescription in both the control and the intervention groups $(P<0.001)$ (Table 4).

With regard to the evolution of pharyngitis in 511 cases (94.1\% of all the cases), full clinical recovery rate at the third week was present in 223 of the 274 patients treated with antibiotics (81.4\%) and in 204 of the 237 $(86.1 \%)$ not treated with antibiotics; the differences were not statistically significant. Side effects were slightly higher among patients treated with antibiotics (32 versus 18), with gastrointestinal side effects being the most frequent. Rash was reported in two cases treated with antibiotics. No complications were observed. 
Table 3. Validity of the rapid antigen detection test depending on the number of Centor criteria

\begin{tabular}{|c|c|c|c|c|c|c|}
\hline $\begin{array}{l}\text { Number of } \\
\text { Centor criteria }\end{array}$ & $n$ & $\%$ GABHS & Sensitivity, \% & Specificity, \% & $\begin{array}{c}\text { Positive predictive } \\
\text { value, } \%\end{array}$ & $\begin{array}{c}\text { Negative predictive } \\
\text { value, } \%\end{array}$ \\
\hline 1 & 73 & $0(-)$ & - & 95.9 & - & 95.9 \\
\hline 2 & 87 & $13(14.9)$ & 84.6 & 89.2 & 57.9 & 97.1 \\
\hline 3 & 74 & 19 (25.7) & 94.7 & 94.5 & 85.7 & 98.1 \\
\hline 4 & 42 & 17 (40.5) & 88.2 & 100.0 & 100.0 & 92.6 \\
\hline Total & 276 & 49 (17.8) & 89.8 & 93.8 & 75.9 & 97.7 \\
\hline
\end{tabular}

GABHS = group $A$ beta-haemolytic streptococcus.

\section{DISCUSSION}

\section{Summary}

The use of RADTs as an aid to primary care physicians in deciding whether adults with acute pharyngitis should be treated with antibiotics is effective in significantly decreasing antibiotic prescription by more than 20\%, and in reducing the inappropriateness of prescription by $33 \%$ in comparison with the group of physicians using only clinical criteria to guide prescription.

\section{Strengths and limitations}

The centres and practitioners participating in this study may have been more motivated than others and may have been more likely to follow current guidelines and follow better clinical practice. A potentially significant limitation is the Hawthorne effect, which may have introduced bias because GPs in the control group may have altered their habits of prescribing. To reduce this risk, GPs in the control group were specifically instructed to follow their usual care. Including a placebo RADT could have reduced this bias but would have been complicated in a study with these characteristics, and it was therefore decided not to do so. In any case, this aspect may have reduced the impact of the results obtained in relation to the optimisation of treatment of acute pharyngitis with RADT. Another limitation is the response rate

Table 4. Inappropriateness of antibiotic prescription by group, according to number of Centor criteria

\begin{tabular}{|c|c|c|c|}
\hline $\begin{array}{l}\text { Number of } \\
\text { Centor criteria }\end{array}$ & $\begin{array}{l}\text { Intervention group } \\
\qquad(N=271), n(\%)\end{array}$ & $\begin{array}{l}\text { Control group } \\
(N=255), n(\%)\end{array}$ & $\begin{array}{c}\text { Total } \\
(N=526), n(\%)\end{array}$ \\
\hline 1 & $4(5.9)$ & $24(39.3)$ & $28(21.7)$ \\
\hline 2 & 20 (23.3) & $48(60.0)$ & $68(41.0)$ \\
\hline 3 & 32 (43.2) & $49(69.0)$ & 81 (55.9) \\
\hline 4 & $17(39.5)$ & $32(74.4)$ & 49 (57.0) \\
\hline Total & 73 (26.9) & $153(60.0)$ & $226(43.0)$ \\
\hline
\end{tabular}

achieved. Even though all the centres agreed to participate, a total of 19 physicians declined. Nonetheless, given the number of cases included in this study, the authors do not believe that this limitation affected the validity of the results obtained.

Another limitation is that all the cases with pharyngitis with at least one Centor criterion were considered. The guidelines currently available recommend the use of RADT in patients with at least two Centor criteria, not those with only one. However, this study was initiated in 2008 when no clear recommendations on the use of RADT were available in Spain. In this study the unit of randomisation was the centre not the physician to avoid contamination among physicians working in the same centre. It was considered that it may have not been appropriate to randomise individuals as patients who attend the same primary healthcare centre may be treated in the same way. None of the physicians included had previously used RADT, so the use of both the RADT technique and pharyngeal swab for microbiological testing were correctly explained beforehand.

\section{Comparison with existing literature}

Few studies have been performed in adults to date that give information on the impact of RADT on antibiotic prescription. Mclsaac et al reported a $45 \%$ reduction in antibiotic prescription in adults using RADT compared with empirical treatment. ${ }^{24}$ Worrall et al reported a proportion of antibiotic prescription of $58 \%$ among physicians who did not use RADTs and $27 \%$ among those who did use this rapid test. ${ }^{20}$ Similarly, in another primary care study carried out in Switzerland, the use of RADT reduced antibiotic prescribing from $60 \%$ to $37 \% .{ }^{23}$ Curiously, in recent studies carried out in paediatrics, in which the incidence of streptococcal infection is higher, greater 
reductions were observed, with percentages of antibiotic prescription among physicians assigned to RADT ranging from $22 \%$ to $28 \% .^{26,27}$

In the present trial, one intriguing question remains about the use of RADT in consulting offices. Even after using RADT, the antibiotic prescription rate exceeded $40 \%$, which is much higher than the known community infection rate with GABHS. The most probable explanation is that physicians assigned to the intervention group did not trust the results of RADT, as more than $30 \%$ of the cases with negative results were prescribed antibiotics. This remained clear in the fact that more than $70 \%$ of the cases with four Centor criteria and a negative RADT result, and almost $60 \%$ with three criteria, received antibiotics. This is probably because physicians in Spain are used to prescribing antibiotic to patients with sore throat and are not used to using rapid tests. Nonetheless, before initiating the study all physicians were informed of the validity of the test and how to perform the technique correctly.

Over recent years, cases of bacteraemic infections caused by Fusobacterium nechrophorum have been reported in young patients, extending the cases of pharyngitis that need antibiotic therapy. ${ }^{28}$ This fact could also explain why so many antibiotics were prescribed with negative test results. This high percentage of antibiotic prescription has also been reported in other studies. In a 6-year retrospective study carried out in Sweden and published recently, negative RADT results were followed by antibiotic prescription in about $40 \%$ of cases. ${ }^{6}$ Similar results were found in a Swiss study, which showed that as many as $75 \%$ of patients presenting a negative test outcome received antibiotics. ${ }^{29}$ Thus, in countries in which rapid tests are still not used it is essential that all GPs are correctly informed of the benefits of this test and the low incidence of streptococcal infection in adults. Moreover, the validity of the test was shown mainly among patients with three or four Centor criteria, and thus, when the RADT result is negative, the physician can trust the result and act accordingly without prescribing an antibiotic. Furthermore, in adults it is necessary to discuss the need indicated by other physicians, mainly paediatricians, to confirm the negative results of the test with a culture, as a negative test discards infection by GABHS in practically all cases.

As expected, side effects were slightly higher among patients treated with antibiotics, with gastrointestinal side effects being most frequent. Of some concern was the finding that, when an antibiotic was used, it was likely to be amoxicillin. This antibiotic, together with the combination of amoxicillin plus clavulanic acid corresponds to broad-spectrum betalactams, which should be avoided in this infection in which it is known that GABHS is uniformly susceptible to penicillin $\mathrm{V}$. In the present study, only $20 \%$ of the antibiotics prescribed corresponded to this antibiotic. Curiously, 20 macrolides were prescribed despite only seven allergies to penicillin being reported. Moreover, the percentages of resistance of GABHS to macrolides are already greater than $15 \%$ of the cases in Spain. ${ }^{30}$

Another finding of note was the specificity recorded by the RADT used in this study, which was $93.8 \%$ when most studies report this to be more than $95 \%$. On the other hand, the sensitivity of the test, at almost $90 \%$, was found to be within the margin described in other studies. ${ }^{22}$ The inclusion of many physicians from 20 different centres may explain the lower specificity. Nonetheless, the authors do not believe that these results should discourage primary care physicians from using RADT in their offices. The main advantage of RADT over throat swab cultures is that the results can be available in only 5 minutes. According to the present results, RADT should not be used if signs of viral infection exist, that is, in those with fewer than two Centor criteria and the use of Centor criteria should only be recommended for select patients who might gain from antibiotic treatment and the use of rapid testing before receiving an antibiotic prescription.

\section{Implications for research and practice}

Based on the results obtained in this study, it would be interesting to carry out qualitative research to investigate the reasons why physicians prescribe antibiotic despite negative test results. This will enable a better approach to this problem, which, according to the results of the present study, is not marginal. The clinical trial could be repeated in other areas to determine whether or not these results are replicated. 
This pragmatic randomised clinical trial found that the use of the RADT in primary care offices was associated with a significant reduction in the prescription of antibiotics among adults with a clinical diagnosis of acute pharyngitis. However, the reduction in antibiotic prescription was expected to be much higher, and this might be mainly explained by the fact that GPs were not used to using these tests and, more specifically, may still be uncertain as to the validity of their results. 


\section{REFERENCES}

1. Neuner JM, Hamel MB, Phillips RS, et al. Diagnosis and management of adults with pharyngitis. A cost-effectiveness analysis. Ann Intern Med 2003; 139(2): 113-122.

2. Llor C, Cots JM, Bjerrum L, et al: grupo de estudio Happy Audit España. Antibiotic prescribing in respiratory tract infections and predictive factors for their use. Aten Primaria 2010; 42(1): 28-35.

3. Patterson CA, Mackeson JM, Weekes LM. Antibiotic prescribing for upper respiratory tract infections in primary care. Commun Dis Intell 2003; 27(suppl): S39-41.

4. Demelker RA, Kuyvenhowen MM. Management of upper respiratory tract infections in Dutch general practice. Br J Gen Pract 1991; 41(353): 507-510.

5. Gonzales R, Steiner JF, Sande MA. Antibiotic prescribing for adults with colds, upper respiratory tract infections and bronchitis, by ambulatory care physicians. JAMA 1997; 278(11): 901-904.

6. Neumark T, Brudin L, Mölstad S. Use of rapid diagnostic tests and choice of antibiotics in respiratory tract infections in primary healthcare - a 6-y follow-up study. Scand J Infect Dis 2010; 42(2): 90-96.

7. Linder JA, Stafford RS. Antibiotic treatment of adults with sore throat by community primary care physicians: a national survey, 1989-1999. JAMA 2001; 286(10): 1181-1186.

8. Cooper RJ, Hoffman JR, Bartlett JG, et al; Centers for Disease Control and Prevention. Principles of appropriate antibiotic use for acute pharyngitis in adults: background. Ann Intern Med 2001; 134(6): 509-517.

9. Pichichero ME, Disney FA, Talpey WB, et al. Adverse and beneficial effects of immediate treatment for group A beta-hemolytic streptococcal pharyngitis with penicillin. Pediatr Infect Dis J 1987; 6(7): 635-643.

10. NICE Short Clinical Guidelines Technical Team. Respiratory tract infections antibiotic prescribing. Prescribing of antibiotics for self-limiting respiratory tract infections in adults and children in primary care. London: National Institute for Health and Clinical Excellence, 2008.

11. Del Mar CB, Glasziou PP, Spinks AB. Antibiotics for sore throat. Cochrane Database Syst Rev 2006; (4): CD000023.

12. Touw-Otten F, Johansen KS. Diagnosis, antibiotic treatment and outcome of tonsillitis: report of a WHO office for 17 European countries. Fam Pract 1992; 9(3): 255-262.

13. Little P, Williamson I. Sore throat management in general practice. Fam Pract 1996; 13(3): 317-321.

14. Petersen I, Johnson AM, Islam A, et al. Protective effect of antibiotics against serious complications of common respiratory tract infections: retrospective cohort study with the UK General Practice Research Database. BMJ 2007; 335(7627): 982.

15. Pichichero ME. Group A streptococcal tonsillopharyngitis: cost-effective diagnosis and treatment. Ann Emerg Med 1995; 25(3): 404-406.

16. Centor RM, Whiterspoon JM, Dalton HP, et al. The diagnosis of strep throat in adults in the emergency room. Med Decis Making 1981; 1(3): 239-246.

17. Gerber MA, Shulman ST. Rapid diagnosis of pharyngitis caused by group A streptococci. Clin Microbiol Rev 2004; 17(3): 571-580.

18. Gerber MA. Comparison of throat cultures and rapid streptococcal tests for diagnosis of streptococcal pharyngitis. Pediatr Infect Dis J 1989; 8(11): 820-824.

19. Mclsaac WJ, Goel V, To T, et al. Effect on antibiotic prescribing of repeated clinical prompts to use a sore throat score: lessons from a failed community intervention study. J Fam Pract 2002; 51(4): 339-344.

20. Worrall G, Hutchinson J, Sherman G, Griffiths J. Diagnosing streptococcal sore throat in adults. Randomized controlled trial of in-office aids. Can Fam Physician 2007; 53(4): 667-671.

21. Bisno AL, Gerber MA, Gwaltney JM, et al. Practice guidelines for the diagnosis and management of group A streptococcal pharyngitis. Clin Infect Dis 2002; 35(2): 113-125.

22. Lasseter GM, McNulty CA. Richard Hobbs F, et al, on behalf of the PRISM Investigators. In vitro evaluation of five rapid antigen detection tests for group A beta-haemolytic streptococcal sore throat infections. Fam Pract 2009; 26(6): 437-444.

23. Humair JP, Revaz SA, Bovier P, Stalder H. Management of acute pharyngitis in adults: reliability of rapid streptococcal tests and clinical findings. Arch Intern Med 2006; 166(6): 640-644.

24. Mclsaac WJ, Kellner JD, Aufricht $P$, et al. Empirical validation of guidelines for the management of pharyngitis in children and adults. JAMA 2004; 291(13): $1587-1595$.

25. Madurell J, Balagué M, Gómez M, et al. Impact of rapid antigen detection testing on antibiotic prescription in acute pharyngitis in adults. FARINGOCAT study: a multicentric randomized controlled trial. BMC Fam Pract 2010; 11(1): 25.

26. Maltezou HC, Tasgris V, Antoniadou A, et al. Evaluation of a rapid antigen detection test in the diagnosis of streptococcal pharyngitis in children and its impact on antibiotic prescription. J Antimicrob Chemother 2008; 62(6): 1407-1412.

27. Ayanruoh S, Waseem M, Quee F, et al. Impact of rapid streptococcal test on antibiotic use in a pediatric emergency department. Pediatr Emerg Care 2009; 25(11): 748-750.

28. Centor RM. Expand the pharyngitis paradigm for adolescents and young adults. Ann Intern Med 2009; 151(11): 812-815.

29. Stürchler MS, Vuille $P$, Semp E, et al. [Diagnosis and antibiotic therapy of infections in outpatients]. Schweiz Med Wochenschr 2000; 130(41): 1437-1446.

30. Pérez-Trallero E, Martín-Herrero JE, Mazón A, et al. Antimicrobial resistance among respiratory pathogens in Spain: latest data and changes over 11 years (1996-1997 to 2006-2007). Antimicrob Agents Chemother 2010; 54(7): 2953-2959. 